\title{
X-ray reflectivity studies of liquid metal and alloy surfaces
}

\author{
M. J. Regan* and P. S. Pershan \\ Division of Applied Sciences and Department of Physics, Harvard University, Cambridge, Massachusetts 02138
}

O. M. Magnussen ${ }^{\dagger}$ and B. M. Ocko

Department of Physics, Brookhaven National Laboratory, Upton, New York 11973

M. Deutsch

Department of Physics, Bar-Ilan University, Ramat-Gan 52100, Israel

L. E. Berman

National Synchrotron Light Source, Brookhaven National Laboratory, Upton, New York 11973

(Received 17 July 1996; revised manuscript received 4 November 1996)

\begin{abstract}
Surface-induced atomic layering at the liquid/vapor interface in liquid metals has been observed using x-ray reflectivity on sputtered clean surfaces under ultrahigh vacuum conditions. A well-defined quasi-Bragg peak is obtained for surfaces of elemental Ga and a Ga-In alloy at large wave vectors $q_{z} \sim 2.3-2.5 \AA^{-1}$. These results are an unambiguous indication of atomic layering with an interlayer spacing $d \sim 2 \pi / q_{z}=2.5-2.7 \AA$. For liquid $\mathrm{Ga}$, the amplitude of the electron-density oscillations, which is significantly underestimated by existing theory and molecular simulation, decays with a characteristic length of $6 \AA$, which is twice that of $\mathrm{Hg}$. Results on the alloy show a clear enrichment of indium at the topmost surface layer, consistent with the Gibbs adsorption rule. The enrichment consists of a single monolayer, with subsequent layers at the bulk eutectic composition. In order to suppress mechanically excited surface waves, the measurements were performed on thin liquid metal films $(<0.5 \mathrm{~mm}$ deep), which leads to a macroscopically curved surface due to the large surface tensions in liquid metals. The experimental challenges posed by measurements on curved surfaces and the techniques that were developed are discussed in detail. [S0163-1829(97)01324-6]
\end{abstract}

\section{INTRODUCTION}

Liquid metal interfaces play an important role in many technologies, from basic metal production processes like smelting and refining processes, where nucleation of gas bubbles and slag/metal reactions are of crucial importance, to more sophisticated metal forming processes like zone melting, casting, and brazing. Each of these technologies are greatly influenced by the unusually high surface tension found in liquid metals which reflects the ordering differences between the surface and bulk. ${ }^{1}$ The details of these differences and, in particular, their temperature dependence near the solid/liquid phase transition are still poorly understood and are at the focus of an extensive research activity over the past decades. ${ }^{2}$ For example, effects like supercooling of a liquid metal below its melting temperature and facet premelting in a metallic crystal were explained in terms of a negative $^{3}$ or positive ${ }^{4}$ surface entropy, respectively, where the reduced entropy in the first case is due to ordering in the liquid at the liquid/crystal interface, and the excess entropy in the second case is due to the reduced dimensionality at the surface. Understanding both the phenomenology and microscopic physics of surface-induced order is very much needed, and although there have been major efforts to understand supercooling and premelting, structural data on the excess order at liquid metal surfaces remain almost nonexistent.

Theory $^{5-10}$ and simulation ${ }^{11-16}$ have suggested that the density normal to the liquid/vapor interface, averaged later- ally over several atomic diameters, of a liquid metal is atomically stratified. Conversely, it is now well established through computer simulation ${ }^{17}$ and theory ${ }^{18,19}$ that the liquid/ vapor interface for simple dielectric liquids follows a simple, monotonic profile. The prediction of layering at liquid metal surfaces is thus a rather remarkable prediction, which distinguishes metallic from dielectric liquid surfaces.

Atomic layering at the surface of a liquid metal can be explained from consideration of the metal as a charged, interacting two-component liquid: a fluid of classical ions that strongly couple via a Coulombic interaction to the conduction electron fluid of Fermi particles. According to Rice and co-workers ${ }^{13}$ the combination of the density dependence of the particle-particle (ion-ion) interactions, the strong Coulomb interactions that try to maintain a local charge neutrality, and the quantum nature of the conduction electrons leads to a situation where the "single-particle" energy density is strongly inhomogeneous. This leads to a sharp transition region from the liquid to its vapor and a well-defined layer of ions at the interface; this topmost layer of ions subsequently introduces layering to the underlying liquid ions, and the "surface-induced" layering extends $\sim 2-4$ atomic diameters into the bulk liquid metal. Although this idea is persuasive, it has proven to be difficult to quantitatively model the disordered liquid metal surface, either in simulations or analytic theories, which is evidenced by the various approaches and approximation different authors have employed over the years. ${ }^{6}$ Furthermore, until recently, surface-induced layering in liquid metals has not been unambiguously demonstrated experimentally. ${ }^{20,21}$ 
Although the interatomic interactions are significantly different for metallic and dielectric liquids, it is interesting to note that simulations show the appearance of atomic layering at the interface between dielectric liquids and a hard wall. ${ }^{17}$ Since surface tensions for liquid metals are much greater than those for dielectric liquids, it is plausible that the differences between the two types of liquid surfaces can be explained in terms of an "effective" hard wall that arises from the specific electron-electron and electron-ion interactions at the surface. Regardless of the particular value of the macroscopic surface tension that governs the excitation of longwavelength capillary waves, it seems likely that excitation of short-wavelength capillary fluctuations on metallic surfaces will be more costly in energy than on dielectric surfaces, and consequently the liquid metal surface will behave like a smooth hard wall that introduces local layering to its topmost layers. Although it is appealing to relate high surface tensions to an effective hard wall, exceptions, such as some alkali metals (e.g., Cs), should not be overlooked. ${ }^{13}$

Several different experimental probes have been employed in the past to study the liquid/vapor interface of metals. Macroscopic experiments (e.g., surface tension, wetting, adsorption, etc.) cannot provide direct microscopic information on the structure of the liquid surface. Scattering experiments, however, can provide subnanometer resolution determination of the structure of the liquid/vapor transition zone. Early optical reflectance and ellipsometry experiments on liquid Hg (Ref. 22) showed the existence of a transition region of subnanometer thickness but lacked the resolution to resolve its internal structure. More recent electron-diffraction measurements were also unable to distinguish between a monotonic and layered density profile model for the interface. $^{23}$ The surface rigidity and corrugation of several liquid metals ${ }^{24}$ was probed using scattering of inert gas atoms. Ga, In, and $\mathrm{Bi}$ were found to have a stiffer and smoother surface than organic liquids, indicating a rather sharp transition zone from liquid to vapor.

With $\mathrm{x}$-ray techniques, it is straightforward in principle to observe surface layering in liquid metals but technically very challenging. To observe layering on a scale defined by the length $d$, the x-ray reflectivity measurements must be extended to wave-vector transfers beyond $q_{2} \sim 2 \pi / d$. If there is layering, as has been observed at some liquid crystal surfaces with $d$ on the order of a few nanometers, ${ }^{25}$ there is a quasiBragg peak in the specular reflectivity at $q_{z} \sim 2 \pi / d$, which is due to the constructive interference of the reflected $\mathrm{x}$ rays from underlying layers. The fact that this peak is confined to the specular condition allows the reflectivity technique to distinguish surface structure from the bulk structure. The difficulties associated with measurements on liquid metals are primarily due to the large $q_{z}$ range $\left(\sim 3 \AA^{-1}\right.$ ) that must be attained to establish atomic layering $(d \sim 2.5 \AA)$ and the consequently low $\mathrm{x}$-ray reflectivity, of order $10^{-9}$ at this large $q_{z}$. It should be noted that X-ray measurements over a simi$\operatorname{lar} q_{z}$ range on simple dielectric liquid surfaces (e.g., water, methanol, ethanol, etc.) are even more difficult and have not yet been achieved. The primary reason for this is that the mean-square amplitude of the thermal roughness due to capillary waves is comparable to the layer spacing. ${ }^{26-28}$ When this is combined with the lower electron density than, for example, $\mathrm{Ga}$ or $\mathrm{Hg}$, the anticipated reflectivity is extremely small and would be very difficult to measure.

Previous measurements by Bosio et al. ${ }^{29}$ and Kawamoto et $a l .{ }^{30}$ on liquid Ga extended to $q_{z} \sim 0.6 \AA^{-1}$ or to a length scale $d \sim 10 \AA$. These measurements confirmed that the liquid/vapor interfacial region is relatively sharp, with no deviations from the average structure extending beyond 10 $\AA$. Although the results of Bosio et al. ${ }^{31}$ could be modeled with an atomically layered density profile, their coarse resolution allowed an equally good modeling by a monotonic profile, as shown by the authors. For $\mathrm{Ga}$, with an atomic diameter $d \sim 2.5 \AA$, the signature of atomic layering is expected to be a peak in the specular reflectivity near $q_{z} \sim 2.4$ $\AA^{-1}$. In addition, Ga readily oxidizes, ${ }^{32,33}$ and ultrahigh vacuum (UHV) and surface cleaning techniques were not employed by Bosio et al. ${ }^{31}$ but have been utilized by Kawamoto et $a l .^{30}$ and Regan et al. $^{21}$ The experiments presented here have relied on UHV conditions and measurements that extend out to $q_{z} \sim 3 \AA^{-1}$ to unambiguously establish surface-induced atomic layering. This approach differs substantially from measurements on $\mathrm{Hg},{ }^{20}$ which are simplified by its relatively small reduction potential that consequently allows the surface to be kept oxide free by enclosing it in a reducing atmosphere of $\mathrm{H}_{2}$ gas. For most other metals this is not possible, and surface studies will require UHV techniques.

We begin in Sec. II with a discussion of the sample preparation procedure, UHV chamber and surface cleaning, liquid spectrometer, and the use of various $\mathrm{x}$-ray techniques to probe curved surfaces. Since results from the elemental Ga surface at room and elevated temperature have been discussed elsewhere, ${ }^{21,34} \mathrm{Sec}$. III provides only a brief summary of the room-temperature results and a general discussion of the atomic layering in Ga. Section IV is devoted to experimental results recorded on the Ga-In eutectic alloy, where surface enrichment effects in the atomic layering can be clearly observed.

\section{EXPERIMENT}

The experimental approach outlined here is based on UHV techniques and sputtering to ensure clean surfaces and X-ray reflectivity data to wave vectors as large as $q_{z}=3.0$ $\AA^{-1}$ to obtain angstrom-scale resolution.

\section{A. Thin liquid samples}

To obtain a stable reflection of $\mathrm{x}$ rays, electrons, etc., from a liquid surface, one must suppress the vibrations that arise from the coupling to equipment vibration and noise. For a shallow liquid $\mathrm{Ga}$ layer ( $\sim 0.3 \mathrm{~mm}$ thick), surface waves with wavelengths significantly greater than $0.3 \mathrm{~mm}$, or with frequencies significantly less than $\sim 1 \mathrm{kHz}$, are attenuated by viscous drag at the liquid/substrate interface. ${ }^{35}$ The UHV chamber and associated goniometers are themselves mounted on an optical table whose resonant frequencies are well below $1 \mathrm{kHz}$. As a consequence, the table serves to attenuate the short-wavelength acoustic disturbances and the viscous drag of the thin film attenuates the long-wavelength excitations.

For the case of liquid $\mathrm{Ga}$ and most other metals, the very high surface tensions and any remnant oxide layer that exists 
at the liquid/substrate interface often prevent wetting from occurring on nonreactive substrates. In most cases, when liquid $\mathrm{Ga}$ is spread on a substrate, it dewets and forms spherical droplets. A procedure was developed ${ }^{36,37}$ to overcome this problem by sputter cleaning the surface of a water-cooled, 1.25-in-diameter Mo substrate in a dc glow discharge of $\mathrm{Ar}$ and then dropping the liquid Ga (99.99999\% purity) through the discharge and onto the glowing Mo. The Ga does not immediately wet the Mo surface but rather tends to form a large droplet, nearly covering the entire surface. With the use of glass wipers, it is straightforward to physically sweep excess Ga from covered spots to the bare regions while maintaining the glow discharge. This method leads to a thin Ga layer $<0.5 \mathrm{~mm}$ thick and contact angles as small as $\sim 10^{\circ}$ (as judged by eye). The glow discharge is then turned off and the chamber vented. The samples are extracted from the glass chamber and then frozen in a nitrogen environment.

\section{B. Ultrahigh vacuum $x$-ray chamber}

Before inserting a sample into the chamber, it is melted and the surface is carefully swept with a clean glass slide to remove any macroscopic oxide particles. The liquid sample is transported into the $\mathrm{x}$-ray UHV chamber by first placing it into a load lock, and then transporting it to the UHV chamber. Surface oxides that form during transport, when the samples are exposed to air, are removed by sputtering with focused 2-keV/Ar ions. ${ }^{30}$ Due to the small sample size and large ion beam cross-sectional area, the surface could be sputtered clean without recourse, to a mechanical wiper. ${ }^{38}$ As noted by others, ${ }^{39}$ the surface impurities that lie outside the local sputtered area readily migrate into the region being cleaned, are broken up, and ultimately completely sputtered off the Ga surface. Additional information and details of the UHV chamber and sample cleaning procedure have been given elsewhere. ${ }^{36}$

\section{Liquid surface spectrometer}

To obtain the high $\mathrm{x}$-ray intensity required for these measurements, the wiggler beamline X-25 at the National Synchrotron Light Source was used. ${ }^{40}$ As a consequence of the high heat load $\left(\sim 200 \mathrm{~W} / \mathrm{mm}^{2}\right)$ of the incident beam on the monochromator, ${ }^{41}$ methods and techniques were developed in the construction and use of a white-beam liquid reflectometer. ${ }^{42}$ We used a single-reflection, water-cooled $\mathrm{Ge}(220)$ crystal to select the $\mathrm{x}$-ray wavelength as well as to deflect the beam downward to a desired angle of incidence $\alpha$ with respect to the horizontal liquid surface. The $\mathrm{x}$-ray energy was set to $18986 \mathrm{eV}(\lambda=0.6532 \AA)$ by rotating the monochromator about a vertical axis and setting it to the $K$-absorption edge of a $\mathrm{Nb}$ foil. An energy resolution of 100 $\mathrm{eV}$ was determined from the width of the absorption edge.

Several combinations of beam divergences and slit settings were tested for these reflectivity studies, since, as will be discussed below, careful control of the beam footprint on the surface is necessary to understand and characterize the influence of sample curvature. Generally, we found that a highly convergent beam, with as small a spot size at the sample as possible, works best. Upstream apertures were used to limit the vertical and horizontal beam divergence, which were set to 0.1 and $0.5 \mathrm{mrad}$, respectively. The dis- (a)
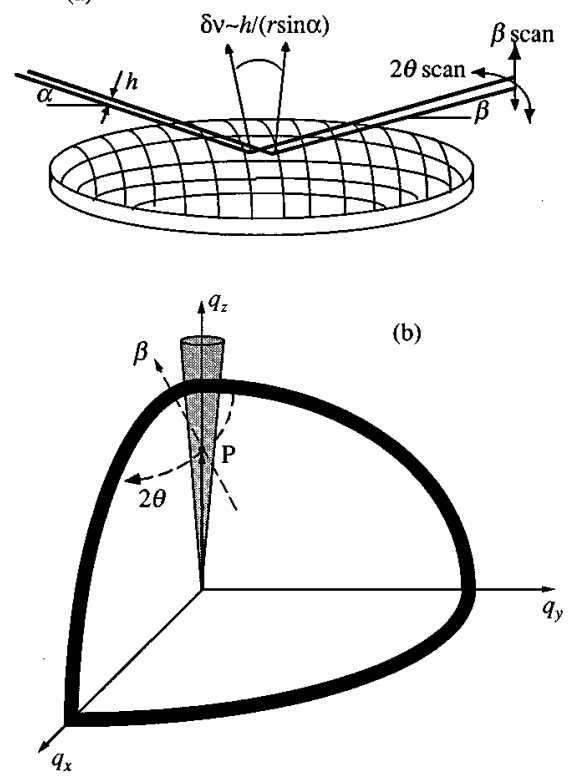

FIG. 1. (a) Schematic diagram of the curved liquid surface kinematics (curvature exaggerated for clarity). The x-ray footprint on the liquid surface strikes the surface where the local normal is at an angle $\nu$ to the vertical, with $r$ the local radius of curvature. To profile the reflection, $\beta$ scans (in the plane of reflection) and $2 \theta$ scans (normal to the plane of reflection) are used. (b) Reciprocal space picture of the specular reflectivity and bulk scattering from a liquid metal. The broad ring represents the isotropic scattering from the first peak in the bulk liquid structure factor. The plane of reflection is the $q_{y}-q_{z}$ plane, and the specular condition is when $q_{x}=q_{y}=0$.

tance between the monochromator crystal and liquid sample is $\sim 600 \mathrm{~mm}$; approximately halfway between the two are the incident slits, which were typically set at $0.07 \mathrm{~mm}$ vertical by $1 \mathrm{~mm}$ horizontal. Immediately downstream of the incident slits is an ion chamber that serves as a monitor of the x-ray flux incident on the sample. Given the beam divergence and location of the incident slits, the beam height at the sample position is typically $h \sim 0.1 \mathrm{~mm}$. The scattering from the sample is recorded with a scintillator detector, $\sim 600 \mathrm{~mm}$ from the sample surface. The spectrometer resolution is determined by a combination of the size of the illuminated spot on the sample and the detector slits, which were typically set at $1 \mathrm{~mm}$ vertical by $4 \mathrm{~mm}$ horizontal.

\section{Reflectivity off curved surfaces}

Although relatively flat liquid metal samples have been obtained, appreciable curvature still exists and complicates the reflectivity method that has been applied successfully in the past to uniformly flat liquid surfaces. A modified method, adapted to curved surfaces, was therefore developed and is described. Figure 1(a) illustrates the curved liquid surface kinematics. The height of the incident beam is $h$, which makes an angle $\alpha$ to the horizontal. Since the transverse width of the beam is small, the sample curvature normal to the reflection plane can be neglected. We first discuss the kinematics of a single ray. Consider the ray that strikes the sample at the highest point where the local normal is vertical. 
By changing the sample vertical position $s$, the position where the beam strikes the liquid surface moves off of the top to a point where the local normal makes an angle $\nu(s)$ to the vertical (within the plane of incidence). The specularly reflected $\mathrm{x}$ ray leaves this point making an angle $\beta=\alpha+2 \nu(s)$ to the horizontal and wave-vector transfer $q_{z} \approx(4 \pi / \lambda) \sin [(\alpha+\beta) / 2]$. Throughout this work, the reference frame is defined so that $q_{z}$ is normal to the curved liquid surface. Thus, the orientation of $\mathbf{q}$, relative to the vertical, depends on the extent of curvature and the location of the incident beam on the curved sample. Since the beam height at the sample position $h$ illuminates a footprint length on the sample of $h / \sin (\alpha)$, the angle of the surface normal will vary by $\delta \nu \approx h /[r \sin (\alpha)]$, where $r$ is the local curvature. Neglecting the small divergence and nonuniformity of the incident beam, the full width at half maximum of the reflected beam will thus be $\Delta \beta \approx h /[r \sin (\alpha)]$.

Consider an incident angle on the order of $3^{\circ}$ (nominal $q_{z}=1 \AA^{-1}$ ) with the beam height at the sample position adjusted to be typically $h=0.1 \mathrm{~mm}$ (determined by the incident slit height, beam divergence, and distance from slits to sample). Under these conditions the beam footprint on the curved surface extends $\sim 2 \mathrm{~mm}$ in the plane of incidence. For a drop with a local radius of curvature $r \sim 200 \mathrm{~mm}$, which is typical at the top of the Ga drops studied here, the orientation of the surface normal then varies by $\sim 9.5 \mathrm{mrad}$ $\left(0.55^{\circ}\right)$ over the illuminated area. This is almost two orders of magnitude larger than the angular divergence of the incident beam, and aside from setting a practical limitation on the experimental resolution, it presents other complications. Since for an ideal flat surface the reflectivity varies as $1 / q_{z}^{4}$, even small errors in the sample height yield significant variations in reflected signal.

A second difficulty is the subtraction of diffuse scattering from the bulk liquid. Figure 1(b) illustrates the significance of the bulk scattering in reciprocal space. The shaded conical region centered on the $q_{z}$ axis is the locus of points where specular reflection could be observed for a curved sample illuminated with a finite footprint. The broad spherical shell, whose intersection with the coordinate planes is shown in thick black stripes, denotes the first peak in the bulk liquid structure factor $S(q)$. For a given incident angle $\alpha$ the specular reflection is centered at a $q_{z} \approx(4 \pi / \lambda) \sin (\alpha)$. To isolate the specular reflection from the diffuse background, two different scans were used to profile the reflected beam (see Fig. $1)$. The first type is a $\beta$ scan in which the detector height is scanned within the plane of incidence $\left(2 \theta=0^{\circ}\right)$, with the incident angle $\alpha$ fixed. In this case, the scan is entirely in the $q_{y}-q_{z}$ plane, and the specular condition is met for a range of $\beta$ surrounding $q_{y}=0$. For a flat sample this occurs when $\beta=\alpha$. For a curved sample, specular reflection is observed over the range defined by $\beta=\alpha+2 \nu(r)$, where $\nu(r)$ varies across the beam footprint. The other measurement is a $2 \theta$ scan, which is a scan through the plane of reflection ( $\alpha$ and $\beta$ fixed). Neglecting sample curvature over the width of the beam, specular reflection for this scan is achieved at $2 \theta=0$, or $q_{x}=0$.

A $\beta$ scan through point $P$ in Fig. 1(b) cuts through the shaded region at an angle and gives rise to a spectrum like that shown in Fig. 2(a) for $\alpha=2 \cdot 3^{\circ}$. The broken line in Fig.
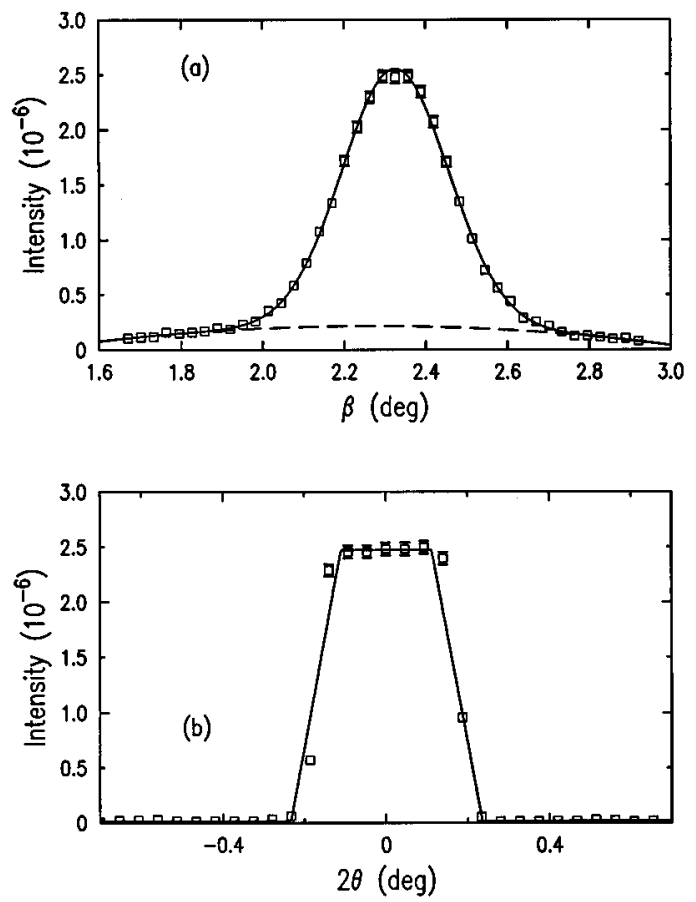

FIG. 2. (a) $\beta$ scan for a small incident angle ( $\alpha=2.3^{\circ}, q_{z}=0.782 \AA^{-1}$ ) where there is little bulk scattering. The dashed line is a fit to the diffuse scattering, and the solid line is the superposition of a Gaussian function and a quadratic background. (b) Corresponding scan through the plane of reflection (scan $2 \theta ; \beta$ set at the specular condition).

2(a) represents diffuse scattering from either bulk scattering or the various $\mathrm{x}$-ray windows of the reflectometer. The local radius of curvature is calculated to be $r \sim 360 \mathrm{~mm}$, which is one of the flattest samples we have obtained (typical values range from $r \sim 100-350 \mathrm{~mm}$ ). Figure 2(b) shows a $2 \theta$ scan through the same point $P$ as illustrated in Fig. 2(a). In this case the only feature of the spectrum that is sensitive to the sample curvature is the peak height which, in absolute units, is identical to that of the $\beta$ scan of Fig. 2(a), as is indeed observed. The line shape in Fig. 2(b) corresponds to the expected two-slit resolution shape for the known incident and detector slits. For this incident angle, neither the $\beta$ nor $2 \theta$ scans record significant intensity from the diffuse scattering associated with the bulk liquid; however, at larger angles when $P$ approaches the point where the specular and bulk diffuse scattering cross, the $\beta$ and $2 \theta$ scans are essential for separation of their relative contributions to the measured intensity. This will be discussed below in connection with Fig. 4.

To ensure that measurements were obtained on the topmost and therefore flattest region of the sample, the $\beta$ scans were recorded for a series of sample heights $s$ for a given $\alpha$, thereby moving the beam footprint systematically across the Ga surface and sampling regions of different local curvature. For each height $s$ the individual $\beta$ scans have been fit to a model consisting of a Gaussian peak ${ }^{43}$ and quadratic background. The form for the Gaussian peak, which represents the intensity distribution of the specular reflection $\left[I_{\beta \text {-scan }}(\beta)\right]$, is thus modeled as 


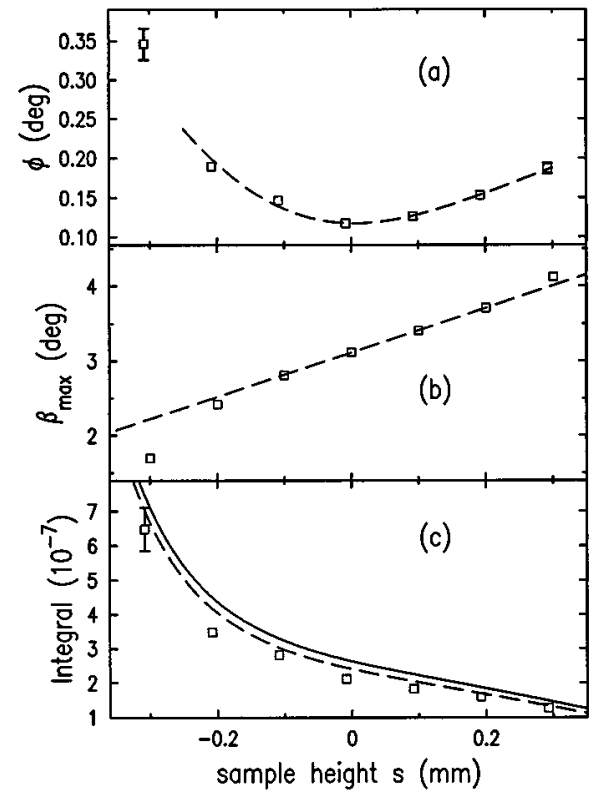

FIG. 3. Illustration of the analysis of $\beta$ scans as a function of sample height $s$ for $\alpha=3.1^{\circ}$, as discussed in text. (a) Gaussian width of the central peak $\phi(s)$; (b) position of reflection $\beta_{\max }(s)$ compared to that expected for constant curvature (dashed line); and (c) integrated intensity compared to Fresnel theory (solid) and model (dashed).

$$
I_{\beta \text {-scan }}(\beta)=\frac{I(s) \Omega_{\text {res }}}{\sqrt{2 \pi} \phi(s)} \exp \left[-\frac{\left[\beta-\beta_{\max }(s)\right]^{2}}{2 \phi(s)^{2}}\right],
$$

where $\phi(s)=\Delta \beta(s) /[2 \sqrt{2 \ln (2)}]$ is the Gaussian width in degrees and $\beta_{\max }(s)$ is the peak position. In view of the fact that the measured intensity distribution, $I_{\beta \text {-scan }}$, is actually a convolution of the differential scattering cross section with a resolution function, the reflectivity is obtained by either digitally integrating the scan or otherwise performing a deconvolution that would relate the reflectivity to the peak of $I_{\beta \text {-scan }}$. In our case, the sample curvature leads to a width $\phi(s)$ that is much broader than the resolution, $\Omega_{\text {res }}=(180 / \pi)\left(D_{\operatorname{det}} / L_{\text {det }}\right.$ ) (in degrees) with $L_{\text {det }} \sim 600 \mathrm{~mm}$ the distance from the sample to detector. As a result, the deconvolution can be performed trivially, with the measured intensity related to the absolute reflectivity, $I(s)$, through multiplication by $\Omega_{\text {res }}$, as shown in Eq. (1).

Figure 3 illustrates the results of these fits for varying sample height and an incident angle $\alpha=3.1^{\circ}$. The fact that the Gaussian width $\phi$ varies with sample height illustrates that the sample curvature is not constant (to first order in $s)$. The local curvature $r(s)$, calculated from $\Delta \beta$ and the footprint length, is $350 \mathrm{~mm}$ at the top of this Ga drop and decreased to $225 \mathrm{~mm}$ for distances up to $\pm 1 \mathrm{~cm}$ from the center. To first order, the average radius of curvature $r_{c}$ is obtained from Fig. 3(b) as the slope, $d \beta_{\max } / d s \approx 1 /\left(r_{c} \alpha\right)$, which leads to $r_{c} \sim 350 \mathrm{~mm}$ at the drop of the top. There is good agreement between the local radius of curvature and the average curvature. Although $\phi(s)$ and $\beta_{\max }(s)$ depend on the curvature, its background-subtracted integral does not. The reflectivity $R$ for a given $q_{z}(\alpha, s)=(4 \pi / \lambda) \sin [\{\alpha$ $\left.\left.+\beta_{\max }(s)\right\} / 2\right]$, is then the integral $I(s)$, normalized to the in-
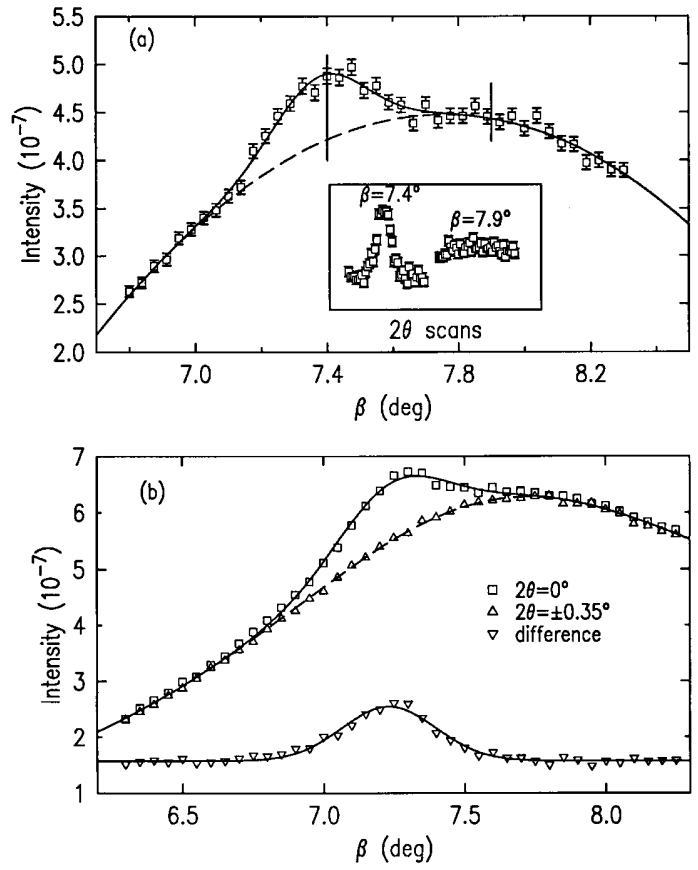

FIG. 4. (a) Illustration of the first method to isolate and quantify the reflectivity in the presence of significant bulk scattering. The broad peak is the first peak in the bulk liquid structure factor, and the smaller peak is from the reflection of $\mathrm{x}$ rays from the liquid surface $\left(\alpha=7.4^{\circ}\right)$. The $2 \theta$ scans illustrate that for $\beta=7.9^{\circ}$ the scattering is isotropic (bulk), and for $\beta=7.4^{\circ}$ the scattering is made up in part by a component confined to the plane of reflection. The vertical lines mark the position in $\beta$ where the $2 \theta$ scans of the inset were taken. (b) Illustration of a second method to isolate and quantify the reflectivity in the presence of significant bulk scattering. The difference between the data measured in the plane of reflection and that offset by $2 \theta= \pm 0.35^{\circ}$ and averaged leads to a well-defined peaked function that is modeled here as a Gaussian function. The difference is offset in the intensity by $1.5 \times 10^{-7}$ for clarity.

cident intensity. It is assumed in this analysis that the reflectivity is a slowly varying function over the width of the $\beta$ scan. The data in Fig. 3(c), obtained from the integrated area of the Gaussian, agrees reasonably well with the calculated Fresnel reflectivity for the measured values of $\alpha$ and $\beta_{\max }$ (solid line); however, the dashed line with somewhat better agreement is obtained from the model reflectivities (discussed below).

The analysis is more subtle at larger values of $q_{z}$ since diffuse scattering from the bulk liquid becomes appreciable and even dominant. Figure 4(a) shows the result of a $\beta$ scan at $\alpha=7.4^{\circ}$, where the specular signal is superimposed on the peak from the bulk scattering, described by the liquid structure factor $S(q)$. Since $S(q)$ is isotropic, as illustrated by the heavy lines in Fig. 1(a), whereas the reflection signal is confined to the line $\mathbf{q}=\left(0,0, q_{z}\right)$, the obvious method to distinguish the specular reflection from $S(q)$ is to move the detector off the specular condition, normal to the plane of reflection, by several resolution widths. In this case, the isotropic bulk scattering is essentially unchanged, whereas the reflection strongly depends on the orientation of $\mathbf{q}$. The inset to Fig. 4(a) illustrates that it is straightforward to discern $S(q)$ from the reflection by utilizing $2 \theta$ scans. When the 

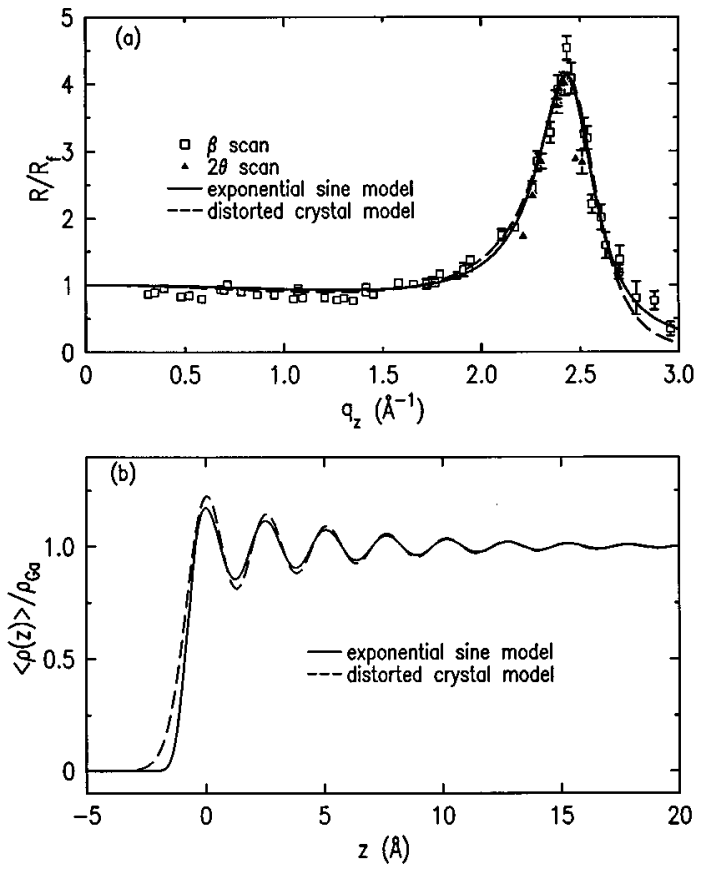

FIG. 5. (a) Reflectivity for liquid gallium normalized by the Fresnel reflectivity: $\square$, from integrating the $\beta$ scans, $\boldsymbol{\Delta}$, from a relative comparison with $2 \theta$ scans. (b) Corresponding electron density profiles for liquid gallium at room temperature.

detector is moved from the specular condition $\beta=7.4^{\circ}$ to the off-specular condition $\beta=7.9^{\circ}$, all of the measured intensity is due to the first peak in the bulk $S(q)$. Consequently, the $2 \theta$ scan yields only a slightly curved background, with little, if any, surface scattering. Conversely, when the detector is in the position to observe specular reflection, the $2 \theta$ scan $\left(\beta=7.4^{\circ}\right)$ results in a resolution limited peak above a similarly curved background. By comparing the relative amplitude changes in the $2 \theta$ scans while systematically varying $\alpha$ and $s$, one can obtain quantitative information about the strength of the reflection as a function of $q_{z}$. Since this method relies on the assumption that the local curvature does not vary over an appreciable range in $s$ (changes in footprint location are less than $5 \mathrm{~mm}$ ), it has been used only in the presence of strong bulk scattering where the first peak in $S(q)$ significantly overlaps the specular peak in the $\beta$ scans.

In this study, most of the reflectivity data were obtained by carrying out $\beta$ scans through the specular condition and at displaced $2 \theta$ as shown in Fig. 4(b). At $2 \theta= \pm 0.35^{\circ}$ the detector does not accept any specular signal and the $\beta$ scan measures only the bulk diffuse scattering, which is essentially constant for small variations in $2 \theta$. Consequently, the independently measured data for the bulk scattering at small offset angles in $2 \theta$ was used to carefully measure and subtract the bulk scattering background in a $\beta$ scan. Figure 4(b) illustrates the results from a $\beta$ scan, both in the plane of reflection and offset by $2 \theta= \pm 0.35^{\circ}$. The difference is the surface signal, which we have modeled as a Gaussian peak superimposed on a linear background. ${ }^{43}$ For this sample, $\Delta \beta$ indicates that $r \sim 150 \mathrm{~mm}$ as compared to $r \sim 350 \mathrm{~mm}$ calculated for the Ga sample in Fig. 4(b); however, the integrated intensities are in good agreement [Fig. 5(a)].

Finally, it should be mentioned that it is difficult to obtain data at small incident angles where the footprint extends significantly on the curved surface. In this case, the x-ray footprint samples regions of curvature that, at one end of the footprint, lead to incident angles close to the critical angle and at the other end may be 5-10 times the critical angle. The constant curvature hypothesis is no longer valid, which complicates the analysis. The low-angle method of Kawamoto et al. ${ }^{30}$ was in fact used, but the best solution would be to obtain a large, flat sample where careful low-angle measurements can be directly obtained.

\section{PURE GALLIUM}

A compilation of the reflectivity data that have obtained for several liquid Ga samples is illustrated in Fig. 5(a). For clarity, the data have been normalized by the theoretical Fresnel reflectivity $\left(R_{f}\right)$ of a perfectly sharp step-function interface and show no appreciable deviation from Fresnel theory except for $q_{z}>2.0 \AA^{-1}$, where a well-defined maximum is evident. At large wave vectors where the bulk scattering is appreciable, there is good agreement of data collected from both the $\beta$ and $2 \theta$ scans. Aside from the known bulk electron density $\rho_{\infty}$, the ratio $R / R_{f}$ can be generally described by as few as four parameters in real space which describe the amplitude, decay length, and spacing of the electron density oscillations into the bulk liquid, and the interfacial roughness. These data have been the subject of a previous publication, ${ }^{21}$ and only a brief summary with further clarification of the mathematical details is presented here. Although a number of density models can be constructed with additional parameters that lead to even better agreement with the data, without additional data at large $q_{z}$, it is not possible to determine much more than the basic features of the layering profile.

For $q_{z}$ larger than 4-5 times the critical wave vector $q_{c}$, where $q_{c}=0.0483 \AA^{-1}$ for gallium, $R / R_{f}$ is related to the average electron density along the surface normal, $\langle\rho(z)\rangle$, by $^{44}$

$$
\frac{R\left(q_{z}\right)}{R_{f}\left(q_{z}\right)} \approx\left|\frac{1}{\rho_{\infty}} \int \frac{d\langle\rho(z)\rangle}{d z} e^{i q_{z} z} d z\right|^{2} .
$$

Typically, the density profile is constructed from a physical model for $\langle\rho(z)\rangle$, inserted in Eq. (2), and fitted to the reflectivity to extract the parameters which best describe the data. Two levels of modeling are discussed here. The first is a simple continuous model for the density, treating the density oscillations and the liquid-vapor transition width on a purely phenomenological level without reference to its atomistic nature. On a more fundamental level, a second model considers the atomic nature of matter, and is based on a detailed atomic description of the layered atoms and the atomic form factor.

A simple continuous model, similar to previous ones used for liquid crystal surfaces ${ }^{25}$ is based on an error-function interfacial profile (width $\sigma$ and offset by $z_{0}$ ) modulated by an exponentially decaying sine wave:

$$
\frac{\langle\rho(z)\rangle}{\rho_{\infty}}=\frac{1}{2}\left(1+\operatorname{erf}\left[\frac{z-z_{0}}{\sigma}\right]\right)+\theta(z) A \sin (2 \pi z / d) e^{-z / \xi}
$$


$\theta(z)$ is the Heaviside step function, $d$ the interlayer spacing, $\xi$ the exponential decay length, and $A$ an amplitude. It is straightforward to compute the reflectivity for this model:

$$
\frac{R\left(q_{z}\right)}{R_{f}\left(z_{z}\right)}\left|\exp \left(i q_{z} z_{0}-\frac{\sigma^{2} q_{z}^{2}}{4}\right)+\frac{4 \pi q_{z}^{2} A}{\xi d D}-i \frac{2 \pi q_{z} A C}{d D}\right|^{2},
$$

with $C=\left(1 / \xi^{2}+4 \pi^{2} / d^{2}-q_{z}^{2}\right)$ and $D=C^{2}+4 q_{z}^{2} / \xi^{2}$. The best-fit profile is shown in Fig. 5(a) (solid line), with $d=2.56 \pm 0.01 \quad \AA, \quad \xi=5.8 \pm 0.4 \quad \AA, \quad A=0.20 \pm 0.02$, $\sigma=0.50 \pm 0.04 \AA$, and $z_{0}=-0.24 \pm 0.06 \AA .{ }^{45}$ The electrondensity profile is illustrated in Fig. 5(b); density profiles computed within the error bars of the parameters are indistinguishable in the figure.

Similar results are obtained with the distorted crystalline model. ${ }^{20,21,34}$ This model assumes basically a layer ordered structure, where the surface layers become increasingly disordered with distance from the surface plane (i.e., the rootmean displacement of the layers $\sigma_{j}$ increases with depth into the bulk liquid as $\sigma_{j}^{2}=\sigma_{\mathrm{cw}}^{2}+j \bar{\sigma}^{2}$ with $\bar{\sigma}$ a measure of the increasing root-mean displacement as the density approaches the bulk liquid and $\sigma_{\mathrm{cw}}$ a displacement common to each layer). With the spacing $d$ between layers fixed, the electron density profile is

$$
\langle\rho(z)\rangle=\frac{n_{\mathrm{Ga}} d}{\sqrt{2 \pi}} \sum_{j=0}^{\infty} \frac{1}{\sigma_{j}} \exp \left[\frac{-(z-j d)^{2}}{2 \sigma_{j}^{2}}\right] \otimes f_{\mathrm{Ga}}(z),
$$

where $n_{\mathrm{Ga}}$ is the number density of atoms in bulk liquid Ga and the Gaussian constructed layered profile is convoluted (i.e., $\otimes$ ) with the $\mathrm{Ga}$ atomic density distribution, $f_{\mathrm{Ga}}(z)$. The reflectivity is

$$
\frac{R\left(q_{z}\right)}{R_{f}\left(q_{z}\right)}=\left|\frac{f\left(q_{z}\right) q_{z} d}{Z}\right|^{2} \frac{e^{-q_{z}^{2} \sigma_{\mathrm{cw}}^{2}}}{\left|1-e^{i q_{z} d-q_{z}^{2} \bar{\sigma}^{2} / 2}\right|^{2}},
$$

with $Z$ the atomic number and $f\left(q_{z}\right)$ the $\mathrm{Ga}$ atomic form factor. Figure 5(b) shows the best fit (dashed line) of this model: $\quad d=2.51 \pm 0.01 \AA \AA \quad \sigma_{\mathrm{cw}}=0.79 \pm 0.01 \quad \AA, \quad$ and $\bar{\sigma}=0.39 \pm 0.01 \AA^{45}$

Analysis with either model leads to similar electron density profiles [Fig. 5(b)] and essentially the same results. The interlayer spacing $d$ is $\sim 10 \%$ less than the near-neighbor spacing in the bulk liquid, which is expected from the stacking of neighboring layers, and is consistent with nearneighbor distances in crystalline $\mathrm{Ga}$. The layering extends an exponential decay length of $\sim 6 \AA$ into the bulk liquid which corresponds to $\sim 3$ atomic diameters. This is approximately twice the decay length that has been measured for liquid $\mathrm{Hg}$ at room temperature. ${ }^{20}$ Although the origin of the difference is not understood, it may be a result of a reduced ordering tendency of $\mathrm{Hg}$, manifested by the higher vapor pressure and lower surface tension, or an increased ordering at the liquid Ga surface that is manifested in the relatively high degree of covalent bonding which is observed in bulk liquid Ga. ${ }^{46-48}$

For the distorted crystal model, the electron density profile can be interpreted as a local structure that is broadened by thermally induced capillary waves, denoted by the common displacement $\sigma_{\mathrm{cw}}$. Recent experiments on the temperature dependence of the layering for liquid $\mathrm{Ga}$ indicate that the prominent layering peak at $q_{z}=2.4 \AA^{-1}$ decreases dramatically upon heating from room temperature to $170{ }^{\circ} \mathrm{C}$. ${ }^{34}$ The peak width, however, stays unchanged, indicating that the layering decay length is temperature independent. The origin of the change can be fully accounted for by the temperature dependence of capillary-wave-induced surface roughness.

The capillary contribution is determined by balancing the thermal energy $k_{B} T$ with the surface tension $\gamma$ and gravity. The temperature dependence of the capillary width follows a simple form,

$$
\sigma_{\mathrm{cw}}^{2}=\sigma_{0}^{2}+\frac{k_{B} T}{2 \pi \gamma} \ln \left(\frac{k_{\max }}{k_{\min }}\right),
$$

with $\sigma_{0}$ an intrinsic contribution. ${ }^{18,19}$ The logarithmic term in Eq. (7) arises from integration of the $1 / k^{2}$ dependence in the thermal expectation value of the mean-square amplitude of a thermal capillary wave of wave vector $\mathbf{k}$. For infinitely sharp angular resolution $\Delta \beta$ and an ideal flat surface, the shortand long-wavelength cutoffs, $k_{\max }$ and $k_{\text {min }}$, respectively, are determined by the molecular size $d\left(k_{\max }=\pi / d=1.26 \AA^{-1}\right)$ and gravity $\left(k_{\min } \propto \sqrt{\Delta \rho g / \gamma}\right.$ with $\Delta \rho$ the mass density difference between the liquid and vapor). However, for the present $\mathrm{x}$-ray measurements carried out on a curved sample, the smallest wave vectors that can be measured are limited by sample curvature, $k_{\min }=\Delta \beta q_{z} / 2$. Since $\Delta \beta$ is determined by the local radius of curvature $(r \sim 150 \mathrm{~mm})$, the beam height at the sample position $(h \sim 0.1 \mathrm{~mm})$, and incident angle $\alpha$, this leads to $k_{\min }=2 \pi h /(r \lambda)=0.0064 \AA^{-1}$, independent of $q_{z}$. From the Ga surface tension ${ }^{49}$ and the experimental resolution, we calculate the capillary wave contribution with $\sigma_{0}=0.37 \pm 0.027 \AA$, and the other parameters, $d$ and $\bar{\sigma}$, are unchanged. The reflectivity data are well described over the entire measured temperature range (i.e., up to $170{ }^{\circ} \mathrm{C}$ ). ${ }^{34}$ These results indicate that even on an atomic length scale the liquid metal surface is extremely flat with a measurable roughness that is fully accounted for by the broadening expected from capillary wave theory.

Finally, we note that our results are in qualitative agreement with computer simulations which predict a similarly layered profile that extends 3-4 atomic diameters into the bulk. ${ }^{11}$ However, the measured layering amplitudes are significantly underestimated by existing theory and simulation. Comparison of the results with theory, and in particular with the simulations done for gallium and other liquid metals, is complicated by the fact that molecular simulations are performed on relatively small model systems that necessarily cut off the long-wavelength capillary waves. In order to make the comparison with any particular simulation, we have used the measured parameters $\left(d, \sigma_{0}, \bar{\sigma}\right)$ that best fit the Ga data along with a long-wavelength cutoff $k_{\text {min }}$ given by $\pi / L$ where $L$ is the length scale of the particular simulation. When the comparison is made in this way, the amplitudes of the resultant surface oscillations from our measurement are significantly larger than that obtained by theory ${ }^{5-10}$ or simulations ${ }^{11-16}$ for any liquid metal investigated, except Hg. ${ }^{12}$ The simulations for gallium itself underestimate the measured amplitudes by a factor of $2 .{ }^{11}$ Although the amplitudes of the predicted surface oscillations for liquid $\mathrm{Hg}$ are comparable to what we observe for $\mathrm{Ga}$, they are not consis- 
tent with the measured amplitudes for $\mathrm{Hg}$, which are $50 \%$ smaller than that for Ga. Further details are given in Ref. 34 .

\section{LIQUID Ga-In EUTECTIC}

We have also applied these $\mathrm{x}$-ray techniques to investigate atomic layering and surface enrichment at the surface of a liquid metal alloy. Measurements were obtained on the liquid Ga-In alloy at the eutectic composition (16.5 at. \% In; melting point at $15.7^{\circ} \mathrm{C}$ ). Other groups have also utilized $\mathrm{x}$-ray scattering and reflectivity methods in the study of very dilute concentrations of $\mathrm{Bi}$ in liquid $\mathrm{Ga}^{50,51} \mathrm{Gibbs}^{52}$ showed that any component that lowers the surface energy will necessarily segregate to the surface. For an ideal binary mixture, where the chemical interaction between atoms is weak and there is little heat of mixing, the surface enrichment is typically from the component with the lowest surface tension. One would thus anticipate a surface enrichment of In for Ga-In alloy, owing to the $\sim 30 \%$ smaller surface tension of In than $\mathrm{Ga}$ (at the melting points). The surface composition of In atoms, $x_{s}$, can be computed from the Gibbs adsorption rule, where the surface energies between the two components are balanced:

$$
\gamma_{\mathrm{Ga}}+\frac{R T}{A_{\mathrm{Ga}}} \ln \left(\frac{1-x_{s}}{1-x_{b}}\right)=\gamma_{\mathrm{In}}+\frac{R T}{A_{\mathrm{In}}} \ln \left(\frac{x+s}{x_{b}}\right),
$$

with $\gamma_{i}$ and $A_{i}$ the surface tension and molecular areas of the components, respectively, and $x_{b}$ the In concentration in the bulk. For the Ga-In eutectic composition, the prediction for the surface concentration is $\sim 80$ at. \% In. It is well known that there exists a large positive heat of mixing in the liquid and solid Ga-In system, and consequently Eq. (8) underestimates the extent of the In enrichment. ${ }^{53}$ Ion scattering and Auger spectroscopy results support this view indicating that the surface of the eutectic alloy is at least 94 at. \% In. ${ }^{53}$

$\mathrm{X}$-ray reflectivity results are consistent with these observations of an enrichment of In atoms at the surface. Figure 6(a) illustrates the reflectivity results for the Ga-In eutectic alloy at room and at an elevated temperature, along with the Fresnel theory for the eutectic composition. The Fresnel reflectivity is computed using a density for the eutectic that is a linear function of the composition, which is a common assumption. ${ }^{1}$ We have used a number density for liquid $\mathrm{Ga}$ of $n_{\mathrm{Ga}}=0.0527$ atom $/ \AA^{3}$, which is the value at the melting point. Since In melts at $156^{\circ} \mathrm{C}$, it is not clear what value to use for the In density, so we have varied the In number density in the fitting procedures and find that $n_{\text {In }}=0.0376$ atom $/ \AA^{3}$ works well. This is the average of the values computed for liquid In at its melting point $\left(0.0369\right.$ atom $\left./ \AA^{3}\right)$ and for crystalline In $\left(0.0383\right.$ atom $\left./ \AA^{3}\right)$. These values lead to a critical wave vector for the eutectic of $q_{c}=0.0487 \AA^{-1}$. Although the density of some liquid alloys shows a deviation from the linear relationship, it is usually no more than $\pm 4 \%$. ${ }^{1}$ Such small deviations change $R_{f}$ only marginally and do not affect the conclusions drawn here.

The peak in the reflectivity, near $q_{z} \sim 2.3 \AA^{-1}$, is an unambiguous indication of atomic layering at the liquid/vapor interface with an interlayer spacing $d \sim 2.7 \AA$. For the data collected at room temperature, however, there is an important difference from that recorded for elemental Ga. The low

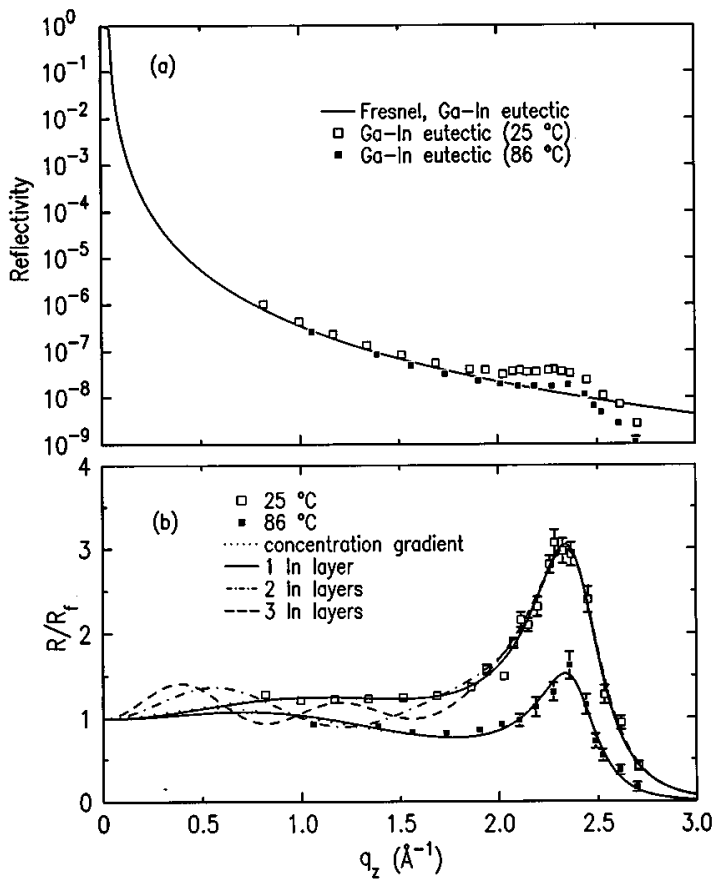

FIG. 6. (a) Measured reflectivity curve for the liquid Ga-In eutectic alloy at room temperature and $86^{\circ} \mathrm{C}$ (all data from $\beta$ scans). The Fresnel reflectivity is denoted by a solid line. (b) Reflectivity data normalized by the Fresnel theory. The data have been fit to the two models discussed in the text, and there is good agreement with a density profile that is layered, with the topmost layer In atoms and the subsequent layers at the Ga-In bulk composition. The fit to the data worsens if additional In layers are included (e.g., with $N=2$ or $N=3$ In layers), so that these are clearly inappropriate.

$q_{z}$ data $\left(<1.5 \AA^{-1}\right)$ are consistently greater than the $R_{f}$ by $\sim 25 \%$, which is an indication that a more dense layer exists on the surface. The models discussed previously cannot fit the data; neither the exponentially decaying sine model nor the distorted crystal model, as constructed, can produce a reflectivity that fits the peak at $q_{z} \sim 2.3 \AA^{-1}$ and still produce a reflectivity greater then $R_{f}$ for $q_{z}<1.5 \AA^{-1}$. However, simple modifications to the distorted crystal model, where segregation of In to the surface can be modeled as an excess density, lead to excellent agreement with the data as shown in Fig. 6(b).

We have pursued two surface segregation models: one with a finite number of In-enriched layers at the surface and the other with a surface enrichment defined by an exponential concentration gradient. To make the discussion clear, we develop each of the models in detail. For an alloy, the density profile can be constructed in terms of the contribution of each element to each of the layers:

$$
\begin{aligned}
\langle\rho(z)\rangle= & \frac{d}{\sqrt{2 \pi}} \sum_{j=0}^{\infty} \frac{1}{\sigma_{j}}\left[n_{\mathrm{Ga}}(j) f_{\mathrm{Ga}}(z)+n_{\mathrm{In}}(j) f_{\mathrm{In}}(z)\right] \\
& \otimes \exp \left[\frac{-(z-j d)^{2}}{2 \sigma_{j}^{2}}\right],
\end{aligned}
$$

with $d$ and $\sigma_{j}$ defined similarly as for pure Ga and $f_{\operatorname{In}}(z)$ and $f_{\mathrm{Ga}}(z)$ the electron density distributions for atomic In and $\mathrm{Ga}$, respectively. The number densities in each layer are 


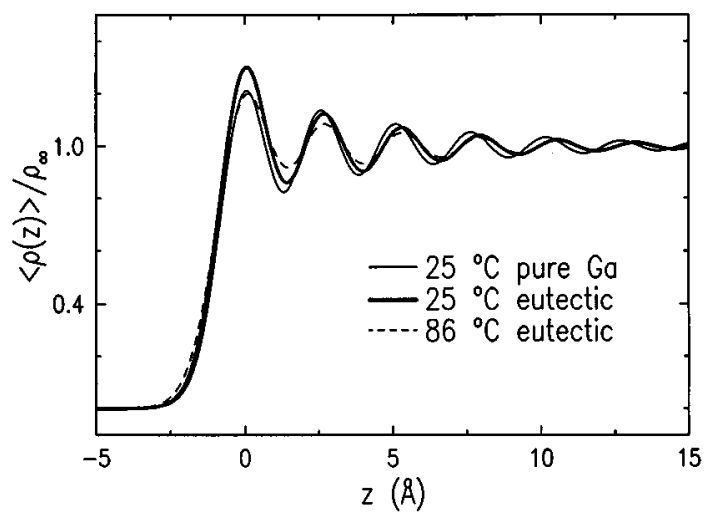

FIG. 7. Models of the electron density profile for the liquid gallium-indium eutectic at room temperature and $87^{\circ} \mathrm{C}$ compared to that for pure gallium. For a given temperature, the two models discussed in the text lead to eutectic density profiles that are indistinguishable in the figure. An enrichment effect in the topmost layer is clearly noticeable.

given by $n_{\mathrm{In}}(j)=x_{j} n_{\mathrm{In}}$ and $n_{\mathrm{Ga}}(j)=\left(1-x_{j}\right) n_{\mathrm{Ga}}$, with $x_{j}$ the concentration of In in the $j$ th layer. Of course it is possible to construct more elaborate models that include additional parameters and are specific to each atom type. The rather simple form of the data, the similarity of the diameters for Ga and In (differ only by $\sim 15 \%$ ), and the good fits that can be accomplished with the present models suggest, however, that more complicated variations of the present model are not necessary here.

As a first example, suppose the top $N$ layers consist of an In-enriched liquid of uniform composition $x_{s}$, with the underlying layers held at the bulk eutectic composition, $x_{b}$. In this scenario, we have

$$
x_{j}=\left\{\begin{array}{l}
x_{s}, j=0,1,2, \ldots, N-1 \\
x_{b}=0.165, \quad j \geqslant N
\end{array}\right.
$$

It is straightforward to compute the reflectivity from Eqs. (9) and (10) in the kinematical approximation [Eq. (2)], assuming as for pure $\mathrm{Ga}$ that the depth dependence of the meansquare displacement of the layers follows a simple form, namely, $\sigma_{j}^{2}=\sigma_{\mathrm{cw}}^{2}+j \bar{\sigma}^{2}$. Figure 6(b) shows the fit of this model to the eutectic data recorded at room temperature, leading to $N=1$ layer, $d=2.59 \pm 0.01 \AA, \bar{\sigma}=0.44 \pm 0.01 \AA$, $\sigma_{\mathrm{cw}}=0.83 \pm 0.01 \AA$, and $x_{s}=0.97-0.11 \pm 0.03$. The fit worsens considerably with additional In-enriched layers (e.g., $N=2$ or $N=3$ ); these can be clearly ruled out, as shown in Fig. 6(b), since they lead to oscillations at small $q_{z}$ which are not evident in the data. Figure 7 shows the density profiles generated from these fits, where there is a density excess in the topmost layer from the In enrichment. It should be mentioned that the value for $x_{s}$ can be varied so that the surface is entirely In or $\sim 86$ at. $\%$ In with little change in the quality of the fits. This is due to the similarity between the In and Ga electron densities, which differ by only $10 \%$. Anomalous reflectivity experiments, which employ the large variation of the dispersion correction to $f(q)$ with energy near an absorption edge, may presumably allow a more accurate determination of the surface composition. Finally, without additional low $q_{z}$ data, one cannot rule out more elaborate surface enrichment models. For example, it is possible to fit the data over the measured $q_{z}$ range to a model with $N=35$ In layers. These models are not physically justified, however, since it is well known from Auger and ion scattering data ${ }^{53}$ that the enrichment does not extend more than a few layers into the bulk liquid. Thus, the profiles that fit the reflectivity data with more than 3-4 In layers can be excluded, but this still needs to be pursued with collection of low- $q_{z}$ data on flat samples.

At higher temperatures $\left(T=86{ }^{\circ} \mathrm{C}\right)$, there is a large decrease in the amplitude of the $R / R_{f}$ ratio, especially near the peak position, with essentially no change in the full width at half maximum. The constant width indicates that the layering decay length is not significantly affected by temperature and the peak height reduction is found to agree with the prediction of capillary wave theory. The same behavior was observed for pure $\mathrm{Ga}$ as well. ${ }^{34}$ The best-fit results at this temperature lead to $N=1, d=2.60 \pm 0.01 \AA, \sigma_{\mathrm{cw}}=0.93 \pm 0.01$ $\AA, \bar{\sigma}=0.40 \pm 0.01 \AA$, and $x_{s}=0.91 \pm 0.17$. The results indicate that for the measured range there is no temperature dependence of the In enrichment, modeled here as the number of In enriched surface layers. Figure 7 illustrates the subtle difference between the two profiles, where the layering at higher temperatures is broadened by the additional displacement from capillary waves, but its $z$ range remains unchanged.

A second model was investigated, also a modification to the distorted crystal model, that led to essentially the same results. The In surface enrichment is modeled as a concentration gradient that starts with a topmost layer of In atoms that decays to the eutectic composition in the bulk over a length scale defined by $\xi$. That is, the concentration in layer $j$ is given by

$$
x_{j}=x_{b}+e^{-j d / \xi}\left(1-x_{b}\right) .
$$

The reflectivity for this model is readily determined from Eq. (2), again assuming $\sigma_{j}^{2}=\sigma_{\mathrm{cw}}^{2}+j \bar{\sigma}^{2}$. Figure 6(b) shows the fits of this model to the eutectic data, which is indistinguishable from the fit to the previous model [Eq. (10)]. The best fit to the data gives $\xi=0.89 \pm 0.92 \AA$, with the other parameters $\left(d, \sigma_{\mathrm{cw}}, \bar{\sigma}\right)$ within the error bars of the previous fit. At 86 ${ }^{\circ} \mathrm{C}$, similar results to the previous model are obtained as well, with $\xi=1.7 \pm 1.2 \AA$, and are indistinguishable in Figs. 6 and 7.

The models lead to the same basic conclusion: only the topmost layer is In rich and the underlying layers are at the bulk eutectic composition, leading to a rather sharp composition change between the $j=0$ and $j=1$ layers. For the concentration gradient model in particular, the decay length is so short that by the $j=1$ layer, the concentration is approximately 21 at. \% In, very close to the 16.5 at. \% In bulk composition. At higher temperatures, we observe that the local layering profile is basically unchanged as in the case of pure $\mathrm{Ga}$, with capillary waves uniformly smearing the profile. Finally, it should be noted that if one models the surface excess as that arising from a monolayer of Ga instead of In, then the fit is considerably worse. In this scenario, since the Ga electron density is smaller at the surface than the density 
of the eutectic bulk, the $R / R_{f}$ for $q_{z}<1.5 \AA^{-1}$ is less than 1 , not greater, as exhibited by the data.

\section{CONCLUSIONS}

In this work, $\mathrm{x}$-ray reflectivity has been used to investigate atomic layering at the free surfaces of liquid $\mathrm{Ga}$ and of the Ga-In eutectic alloy. For both systems, a peak in the reflectivity at large $q_{z}$ has been observed, which is a clear signature of surface-induced atomic layering. For these measurements, special efforts have been made through the use of UHV conditions and sputtering to ensure clean surfaces. We find that the layering extends $\sim 3-4$ atomic diameters into the bulk liquid. In a separate publication we have shown that this penetration into the bulk is insensitive to temperature. ${ }^{34}$ Structural data on the Ga-In eutectic alloy indicate quite clearly an In surface enrichment effect that is restricted to the top monolayer, with the remaining layers at essentially the bulk eutectic composition.

Our results lead to a number of interesting questions that need to be investigated further. For example, it is not clear why the measured amplitudes of the layering oscillations are so much greater than that predicted in analytical theory ${ }^{5-10}$ and simulations. ${ }^{11-13,15,16}$ There are a number of discrepancies between different published theoretical density profiles, both in the amplitude of the oscillations and in the extent of the layering into the bulk liquid, and the present experimental results should help guide future theoretical efforts. In addition it is not clear that the observed layering is ubiquitous to all liquid metals and we have already seen basic differences between the surface profiles of liquid $\mathrm{Hg}$ and both pure $\mathrm{Ga}$ and the Ga-In eutectic. It is therefore important to extend the present meager body of measurements to other liquid metals. Unfortunately, an extension of these measurements to higher melting point metals (e.g., Au, Pt) or those with a relatively small surface tension (e.g., Cs), may not be straightforward. The capillary wave contribution in these cases will be much greater than for $\mathrm{Ga}$ or the Ga-In eutectic. It is not clear if the capillary widths will dominate and completely smear the layering profiles for these metals or if the intrinsic profile will compensate and lead to a strong layering as we observe for $\mathrm{Ga}$ at room temperature.

The methods developed and presented here are applicable to any surface that exhibits a relatively small radius of curvature. With careful tracking of the reflected $\mathrm{x}$-ray beam, as the beam footprint samples different regions of the surface, it is possible to determine the local and average radii of curvature in addition to the local structural characteristics of the surface probed by the $\mathrm{x}$ rays. In general, the methods utilized here can also be extended to other types of measurements (e.g., electron-based methods) of curved surfaces like liquid metals.

\section{ACKNOWLEDGMENTS}

The authors thank E. H. Kawamoto, N. Maskil, and S. Lee for the primary construction of the sample preparation chamber, UHV chamber, and liquid reflectometer, and G. Swislow for assistance with SPEC and monochromator control. This work has been supported by the U.S. Department of Energy Grant No. DE-FG02-88-ER45379, the National Science Foundation Grant No. DMR-94-00396, and the U.S.-Israel Binational Science Foundation, Jerusalem. Brookhaven National Laboratory is supported by U.S. DOE Contract No. DE-ACO2-76CH00016.
*Present address: Hewlett-Packard, IJBU Advanced Research Lab, Corvallis, OR 97330.

†Present address: Abteilung Oberflächenchemie und Katalyse, University Ulm, D-89069 Ulm, Germany.

${ }^{1}$ T. Iida and R. I. L. Guthrie, The Physical Properties of Liquid Metals (Clarendon, Oxford, 1993), p. 109.

${ }^{2}$ O. Tomagnini, F. Ercolessi, S. Iarlori, F. D. DiTolla, and E. Tosatti, Phys. Rev. Lett. 76, 1118 (1996).

${ }^{3}$ D. Turnbull, in Physics of Non-Crystalline Solids, edited by J. A. Prins (North-Holland, Amsterdam, 1964), p. 41.

${ }^{4}$ R. Trittibach, C. Grütter, and J. H. Bilgram, Phys. Rev. B 50, 2529 (1994).

${ }^{5}$ M. Hasegawa, J. Phys. F 18, 1449 (1988).

${ }^{6}$ S. K. Lai, Proc. Nat. Sci. Counc. ROC(A) 15, 191 (1991).

${ }^{7}$ M. A. Gömez and E. Chacön, Phys. Rev. B 46, 723 (1992).

${ }^{8}$ M. Iwamatsu and S. K. Lai, J. Phys. Condens. Matter 4, 6039 (1992).

${ }^{9}$ R. Evans, J. R. Henderson, D. C. Hoyle, A. O. Parry and Z. A. Sabeur, Mol. Phys. 76, 1 (1993).

${ }^{10}$ M. A. Gömez and E. Chacón, Phys. Rev. B 49, 11405 (1994).

${ }^{11}$ Z.-h. Cai and S. A. Rice (unpublished).

${ }^{12}$ M. P. D'Evelyn and S. A. Rice, J. Chem. Phys. 78, 5081 (1983).

${ }^{13}$ J. G. Harris, J. Gryko, and S. A. Rice, J. Chem. Phys. 87, 3069 (1987).

${ }^{14}$ S. A. Rice, Proc. Natl. Acad. Sci. U.S.A. 84, 4709 (1987).

${ }^{15}$ A. Ishida, M. Hasegawa, and M. Watabe, J. Non-Cryst. Solids 117/118, 650 (1990).
${ }^{16}$ S. Iaroli, F. Ercolessi, and E. Tosatti, Surf. Sci. 251, 951 (1991).

${ }^{17}$ G. A. Chapela, G. Saville, S. M. Thompson, and J. S. Rowlinson, J. Soc. Faraday II 73, 1133 (1977).

${ }^{18}$ D. Beysens and M. Robert, J. Chem. Phys. 87, 3056 (1987).

${ }^{19}$ M. P. Gelfand and M. E. Fisher, Physica (Amsterdam) 166A, 1 (1990).

${ }^{20}$ O. M. Magnussen, B. M. Ocko, M. J. Regan, K. Penanen, P. S. Pershan, and M. Deutsch, Phys. Rev. Lett. 74, 4444 (1995).

${ }^{21}$ M. J. Regan, E. H. Kawamoto, S. Lee, P. S. Pershan, N. Maskil, M. Deutsch, O. M. Magnussen, B. M. Ocko, and L. E. Berman, Phys. Rev. Lett. 75, 2498 (1995).

${ }^{22}$ A. N. Bloch and S. A. Rice, Phys. Rev. 185, 933 (1969).

${ }^{23}$ M. Hasegawa and T. Ichikawa, J. Phys. Condens. Matter 3, 2769 (1991).

${ }^{24}$ W. R. Ronk, D. V. Kowalski, M. Manning, and G. M. Nathanson, J. Chem. Phys. 104, 4842 (1996).

${ }^{25}$ P. S. Pershan, A. Braslau, A. H. Weiss, and J. Als-Nielsen, Phys. Rev. A 35, 4800 (1987).

${ }^{26}$ A. Braslau, P. S. Pershan, G. Swislow, B. M. Ocko, and J. AlsNielson, Phys. Rev. B 38, 2457 (1988).

${ }^{27}$ X. Z. Wu, E. B. Sirota, S. K. Sinha, B. M. Ocko, and M. Deutsch, Phys. Rev. Lett. 70, 958 (1993).

${ }^{28}$ B. M. Ocko, X. Z. Wu, E. B. Sirota, S. K. Sinha, and M. Deutsch, Phys. Rev. Lett. 72, 242 (1994).

${ }^{29}$ L. Bosio, R. Cortes, G. Folcher, and M. Froment, J. Electrochem. Soc. 139, 2110 (1992). 
${ }^{30}$ E. H. Kawamoto, S. Lee, P. S. Pershan, M. Deutsch, N. Maskil, and B. M. Ocko, Phys. Rev. B 47, 6847 (1993).

${ }^{31}$ L. Bosio, R. Cortes, A. Defrain, and M. Oumezine, J. Non-Cryst. Solids 61-62, 697 (1984).

${ }^{32}$ J. M. Chabala, Phys. Rev. B 46, 11346 (1992).

${ }^{33}$ M. J. Regan, H. C. Tostmann, P. S. Pershan, O. M. Magnussen, E. DiMasi, B. M. Ocko, and M. Deutsch, Phys. Rev. B 55, 10786 (1997).

${ }^{34}$ M. J. Regan, P. S. Pershan, O. M. Magnussen, B. M. Ocko, M. Deutsch, and L. E. Berman, Phys. Rev. B 54, 9730 (1996).

${ }^{35}$ P. S. Pershan, Faraday Discuss. Chem. Soc. 89, 231 (1990).

${ }^{36}$ E. H. Kawamoto, Ph.D. thesis, Harvard University, 1994.

${ }^{37}$ N. Maskil, Ph.D. thesis, Bar-Ilan University, Israel 1994 (in Hebrew).

${ }^{38}$ G. Indlekofer, P. Oelhafen, and J.-J. Guntherodt, in Physical and Chemical Properties of Thin Metal Overlayers and Alloy Surfaces, edited by D. M. Zehnov and D. W. Goodman, MRS Symposia Proceedings No. 83 (Materials Research Society, Pittsburgh, 1987), Vol. 83, p. 75.

${ }^{39}$ R. G. Musket, W. McLean, C. A. Colmenares, D. M. Makowiecki, and W. J. Siekhaus, Appl. Surf. Sci. 10, 143 (1982).

${ }^{40}$ L. E. Berman, J. B. Hastings, T. Oversluizen, and M. Woodle, Rev. Sci. Instrum. 63, 428 (1992).

${ }^{41}$ L. E. Berman, E. H. Kawamoto, M. Deutsch, B. M. Ocko, and P. S. Pershan (unpublished).
${ }^{42}$ J. Als-Nielsen and P. S. Pershan, Nucl. Instrum. Methods Phys. Res. 208, 545 (1983).

${ }^{43}$ Other models have been used (e.g., Lorentzian function) as well as a numerical integration of the data. Since these methods yield equivalent results, all of the analysis presented here is from modeling with Gaussian functions.

${ }^{44}$ P. S. Pershan and J. Als-Nielsen, Phys. Rev. Lett. 52, 759 (1984).

${ }^{45}$ The error bars reflect the change in the fitted parameter that lead to an increase of $\chi^{2}$ by one standard deviation and do not include possible systematic errors.

${ }^{46}$ P. Ascarelli, Phys. Rev. 143, 36 (1966).

${ }^{47}$ X. S. Gong, G. L. Chiarotti, M. Parrinello, and E. Tosatti, Europhys. Lett. 21, 469 (1993).

${ }^{48}$ A. DiCicco and A. Filipponi, Europhys. Lett. 27, 407 (1994).

${ }^{49}$ K. B. Khokonov, S. N. Zadumkin, and B. B. Alchagirov, Sov. Electrochem. 10, 865 (1974).

${ }^{50}$ E. B. Flom, M. Li, A. Acero, N. Maskil, and S. A. Rice, Science 260, 332 (1993).

${ }^{51}$ N. Lei, Z. Huang, and S. A. Rice, J. Chem. Phys. 104, 4802 (1996).

${ }^{52}$ J. W. Gibbs, The Scientific Papers of J. Willard Gibbs, Vol. 1 (Longmans, Green, New York, 1906), p. 219.

${ }^{53}$ M. F. Dumke, T. A. Tombrello, R. A. Weller, R. M. Housley, and E. H. Cirlin, Surf. Sci. 124, 407 (1983). 